This section provides information for researchers on clinical trials being in progress in their field throughout the world. The list of trials described herein is by no means inclusive, and the publisher is not responsible for any data given.

Please use the special questionnaire at the end of this section to submit information on a new trial.

The Ongoing Clinical Trials are only available online, free of charge, under www.karger.com/dsu_issues

Oncology

Inflammatory Bowel Disease

Surgical Infection / Sepsis

Miscellaneous

Questionnaire forTrial Submission

(C) 2006 S. Karger AG, Basel 


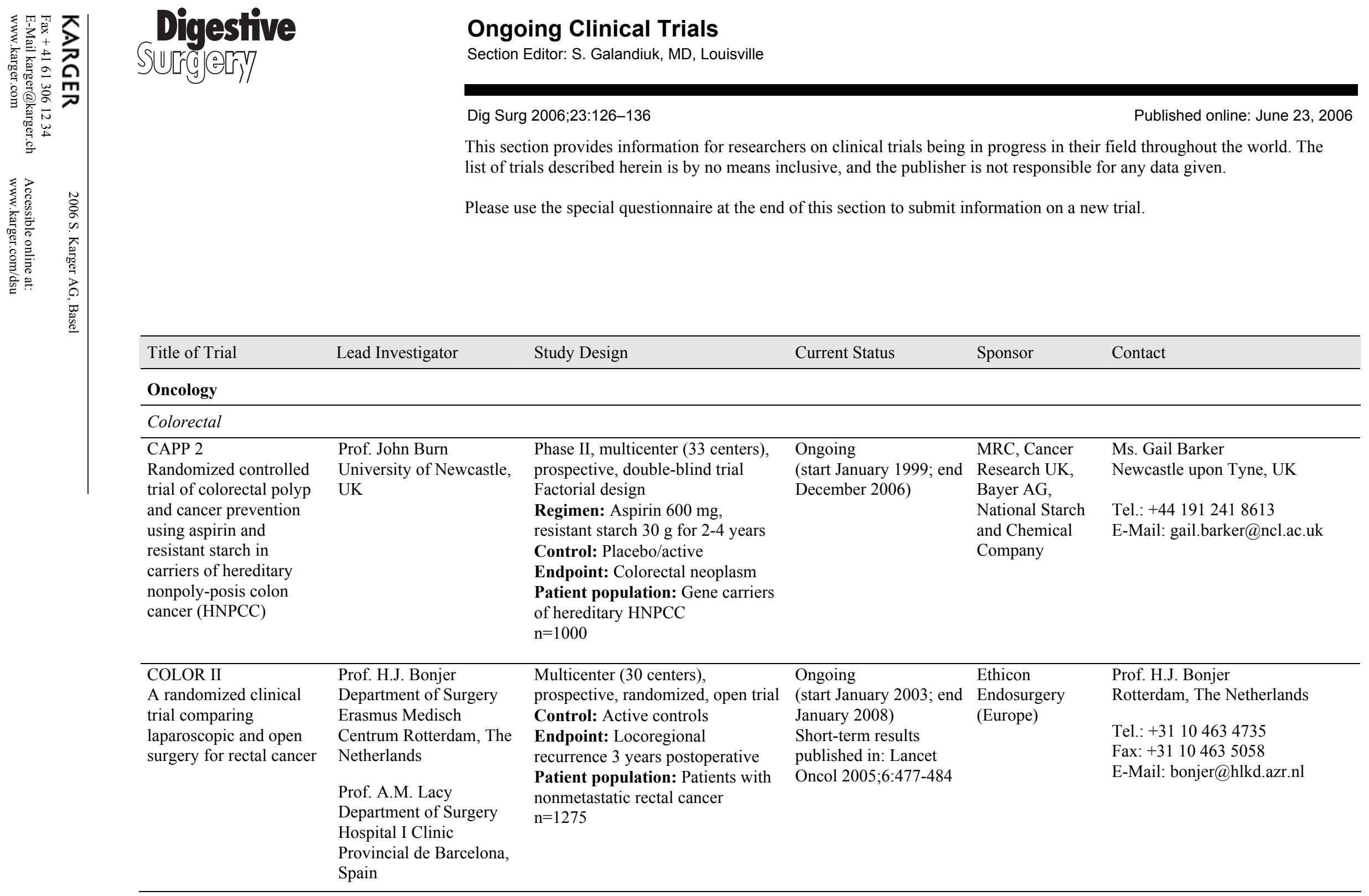


Ongoing Clinical Trials (continued)

\begin{tabular}{lll}
\hline Title of Trial & Lead Investigator & Study Design \\
\hline DaCHS - Prevention of & Prof. Dr. H. Brenner & Independent, case-control trial \\
colorectal carcinoma: & Deutsches Zentrum für & $\mathrm{n}=600$ \\
The role of screening & Alternsforschung, & \\
& Heidelberg, Germany &
\end{tabular}

Sponsor

Contact

Ongoing (start January DZA

2003; end of recruit-

ment December 2007)

(Deutsches

Dr. Christoph Seiler, MS

Heidelberg, Germany

Zentrum für

Altersforschung) Tel.: +496221566986

Fax: +49 6221566988

E-Mail:

christoph_seiler@med.uni-

heidelberg.de

www.sdgc.de

\begin{abstract}
Colon-J-pouch versus
transverse coloplasty

pouch: A randomized

controlled trial com-

paring functional results

after rectum resection

and different recon-

structions
\end{abstract}

ISRCTN No.: 78983587

$\begin{array}{ll}\text { PD Dr. Kaspar Z'graggen } & \text { Multicenter, prospective, ran- } \\ \text { Klinik Beau-Site, Berne, } & \text { domized, open trial }\end{array}$

Klinik Beau-Site, Berne, domized, open trial

Switzerland Control: Comparison of different

techniques

Endpoint: Morbidity, function and

mortality during a follow-up period

of 24 months

Patient population: Patients

showing deep rectum pathology

with planned RO-surgery

$\mathrm{n}=150$

\section{Prospectively random-}

ized study comparing

function and quality of

life after a

coloplasty/low colorect

anastomosis or coloanal

anastomosis versus a

straight anastomosis or a

colonic J-pouch low

colorectal or coloanal

anastomosis

Victor W. Fazio, MD,
Feza Remzi, MD
Cleveland Clinic Foun-
dation, Cleveland, Ohio,

Prospective, randomized trial

Endpoint: 2-year follow up of

functional results

Patient population: Patients with

rectal cancer undergoing elective

surgery

$\mathrm{n}=288$ (72 in each group)

\section{Ongoing}

Dr. Christoph Seiler, MSc

Heidelberg, Germany

Tel.: +49 6221566986

Fax: +49 6221566988

E-Mail:

christoph_seiler@med.uni-

heidelberg.de

www.sdgc.de

Closed; Final data audit
in progress

in progress
in

Cleveland, Ohio, USA

Tel.: +1 8002232273 ext. 54126

E-Mail: zutshim@ccf.org

Dr. Feza Remzi

Cleveland, Ohio, USA

Tel.: +1 8002232273 ext. 54126

E-Mail: remzif@ccf.org

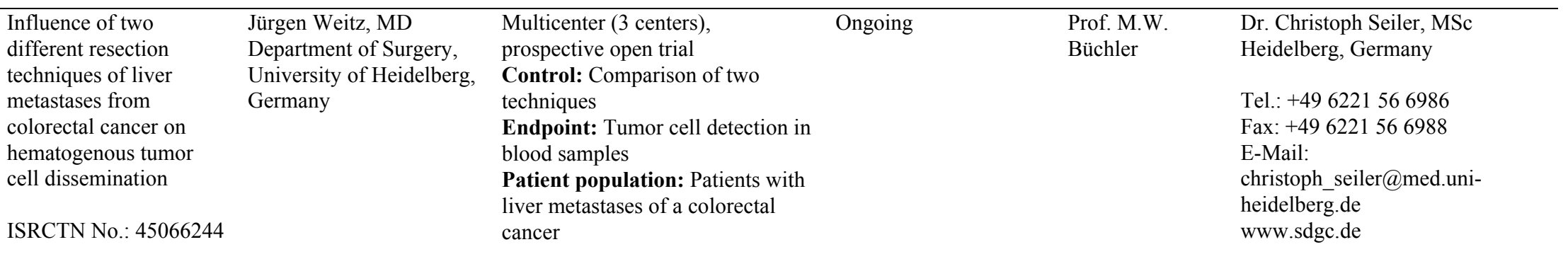


Ongoing Clinical Trials (continued)

\begin{tabular}{|c|c|c|c|c|c|}
\hline Title of Trial & Lead Investigator & Study Design & Current Status & Sponsor & Contact \\
\hline $\begin{array}{l}\text { InTACT } \\
\text { Interdisciplinary trial of } \\
\text { adjuvant colon cancer } \\
\text { treatment }\end{array}$ & $\begin{array}{l}\text { Prof. Dr. W. Hohenberger } \\
\text { University Hospital } \\
\text { Erlangen, Germany }\end{array}$ & $\begin{array}{l}\text { Phase III, multicenter, prospective, } \\
\text { investigator-blind trial } \\
\text { Regimen: } 24 \text {-hour infusion with } \\
\text { 5-FU }\left(2,000 \mathrm{mg} / \mathrm{m}^{2}\right) \text {; folinic acid } \\
\left(500 \mathrm{mg} / \mathrm{m}^{2}\right) \text { once weekly for } 6 \\
\text { months }(\text { adjuvant treatment arm) } \\
\text { Control: Adjuvant therapy versus } \\
\text { surgery (quality controlled) alone } \\
\text { Endpoint: Survival, disease-free } \\
\text { survival } \\
\text { Patient population: Patients with } \\
\text { stage III (UICC) colon cancer } \\
\text { complete resection (R0); } n=240 \\
\text { Additional information: Interim } \\
\text { analysis after randomization of } \\
120 \text { patients }\end{array}$ & $\begin{array}{l}\text { Follow-up } \\
\text { (start October 2001; } \\
\text { end October 2006) }\end{array}$ & & $\begin{array}{l}\text { Dr. Bertram Reingruber } \\
\text { Erlangen, Germany } \\
\text { Tel.: +499131853 } 3296 \\
\text { Fax: +49 } 91318536144 \\
\text { E-Mail: bertram.reingruber@, } \\
\text { stud.uni-erlangen.de }\end{array}$ \\
\hline $\begin{array}{l}\text { ROMIC: Role of ovarian } \\
\text { metastasis in colorectal } \\
\text { cancer }\end{array}$ & $\begin{array}{l}\text { R.M.H. Roumen, MD } \\
\text { Maxima Medical Center } \\
\text { Department of Surgery } \\
\text { Veldhoven, the } \\
\text { Netherlands }\end{array}$ & $\begin{array}{l}\text { Multicenter ( } 12 \text { centers), } \\
\text { prospective, unblinded } \\
\text { (oophorectomy) trial } \\
\text { Control: Patients who do not } \\
\text { undergo oophorectomy } \\
\text { Endpoint: } 3 \text {-year disease-free } \\
\text { survival } \\
\text { Patient population: Postmeno- } \\
\text { pausal women with colorectal } \\
\text { cancer without evidence of } \\
\text { metastatic disease } \\
\text { n=1000 } \\
\text { Additional information: } \\
\text { Incidence of micrometastases in } \\
\text { ovaria of colorectal cancer patients } \\
\text { and prevention of future } \\
\text { Krukenberg tumors }\end{array}$ & $\begin{array}{l}\text { Ongoing (start 2003; } \\
\text { end 2007) }\end{array}$ & & $\begin{array}{l}\text { R.M.H. Roumen, MD } \\
\text { Veldhoven, the Netherlands } \\
\text { Tel.: +31 } 408888556 \\
\text { Fax: +31 } 408888565 \\
\text { E-Mail: r.roumen@mmc.nl }\end{array}$ \\
\hline
\end{tabular}


Pancreatic

Neoadjuvant chemo-

radiation in pancreatic

Prof. Dr. W. Hohenberger Multicenter, prospective,

nonrandomized trial

Ongoing (start Jun

2003; 42 patients

PD Dr. T. Meyer

cancer

Erlangen, Germany

tion: Patients with

as of February

Prof. Dr. G. Grabenbauer the pancreatic head

University Hospital

Erlangen, Germany

$\mathrm{n}=254$

al information: Pilo

project finished, new trial with

gemcitabine and cis-platin as

radiosensitizers

Tel.: +4991318533296

Fax: +4991318536595

E-Mail:

Thomas.meyer@chir.imed.uni-

erlangen.de

PD Dr. T. Brunner

Erlangen, Germany

E-Mail:

thomas.brunner@strahlen.imed.unierlangen.de

\section{GEM-CAP}

Trial comparing

Neoptolemos

Department of Surgery,
University of Liverpool,

combination with

UK

capecitabine for the

advanced pancreatic

ductal adenocarcinoma
Phase III, mulicenter, randomized

tria

Regimen:

Patients treated with gemcitabine

alone or a combination of

gemcitabine and capecitabine for 12

weeks; those responding to

treatment or with stable disease

receive a further 12 weeks of

treatment

Primary endpoint: One-year

survival

Secondary endpoint: Quality of

life; median and two-year survival;

toxicity; objective response rate

Patient population: Patients with

histological or cytological evidence

of locally advanced / metastatic

carcinoma of the pancreas not

amenable to curative surgery

radiotherapy; $\mathrm{n}=508$

\section{closed to recruitment \\ target accrual has been}

reached)

Ms. Claire Davies, Coordinator

Liverpool, UK

Tel.: +44 151794893

Fax. +44 1517948930

E-Mail:cldavies@liverpool.ac.uk 
Adjuvant therapies in

Dr. C.H.J. van Eijck

Phase III, prospective trial; patients Closed

Dr. C.H.J. van Eijck

Erasmus Medisch Centrum randomized into two group

resectable pancreatic Rotterdam, The Control: Surgery alone versus

cancer

Netherlands

intra-arterial chemotherapy and

Rotterdam, The Netherland

radiotherapy

Tel.: +31 104633854

Endpoint: 2-year survival

E-Mail: c.vaneijck@erasmusmc.n

Patient population: $n=120$

\begin{tabular}{|c|c|c|}
\hline $\begin{array}{l}\text { ESPAC-3(v2) } \\
\text { Adjuvant chemo- } \\
\text { therapies in resectable } \\
\text { pancreatic cancer }\end{array}$ & $\begin{array}{l}\text { Prof. J.P. Neoptolemos } \\
\text { Department of Surgery, } \\
\text { University of Liverpool, } \\
\text { UK }\end{array}$ & $\begin{array}{l}\text { International multicenter ( } 130 \\
\text { centers) trial; patients with } \\
\text { pancreatic ductal adenocarcinoma } \\
\text { randomized into one of two groups } \\
\text { (gemcitabine; 5-FU and folinic } \\
\text { acid) and patients with ampullary or } \\
\text { other tumors of the pancreas } \\
\text { randomized into one of three groups } \\
\text { (gemcitabine; 5-FU and folinic acid, } \\
\text { and observation) } \\
\text { Endpoint: } 2 \text {-year survival } \\
\text { Patient population: Patients with } \\
\text { resected pancreatic cancer }\end{array}$ \\
\hline
\end{tabular}

$\mathrm{n}=1030$

Miscellaneous

Multicenter randomized trial for laser-induced

thermotherapy of colo-

rectal liver metastases

Priv. Doz. C.-T. Germer
Department of Surgery,

University Hospital B

Berlin, Germany
Phase III, multicenter (6 centers), randomized trial

Control: Surgical liver resection

Endpoint: Patient survival, quality

of life/mortality

Patient population: Patients with 4

or fewer liver metastases, diameter

$4 \mathrm{~cm}$ or smaller and with no signs of

extrahepatic malignant disease

$\mathrm{n}=400$
Recruitment phase (end Cancer Research Mrs. Emily Owen, Coordinato September 2006)

UK

Liverpool, UK

Tel.: +44 1517948932

Fax: +44 1517948930

E-Mail: e.owen@liverpool.ac.uk

$\begin{array}{ll}\text { Recruitment phase (start BMBF } & \text { Prov. Doz. C.-T. Germer } \\ \text { August 2000; } & \text { Berlin, Germany } \\ \text { end July 2007) } & \text { Tel.: }+493084452543 \\ & \text { Fax: }+493084452740 \\ & \text { E-Mail: germer@ukbf.fu-berlin.de }\end{array}$

E-Mail: germer@ukbf.fu-berlin.de 


\section{Inflammatory Bowel Disease}

\begin{tabular}{|c|c|c|c|c|}
\hline $\begin{array}{l}\text { Multicenter, } \\
\text { randomized, double- } \\
\text { blind, placebo-controlled } \\
\text { retreatment study of } \\
\text { sargramostim } \\
\text { (Leukine } ® \text { ) in patients } \\
\text { with active Crohn's } \\
\text { Disease and prior } \\
\text { treatment response to } \\
\text { sargramostim (Berlex } \\
\text { protocol 308180) }\end{array}$ & $\begin{array}{l}\text { Phase III, multicenter, randomized, } \\
\text { double-blind, placebo-controlled } \\
\text { trial } \\
\text { Regimen: Leukine }{ }^{\circledR} \\
\text { (sargramostim) or placebo } 6 \mathrm{~g} / \mathrm{kg} \\
\text { daily, administered subcutaneously } \\
\text { Control: Placebo } \\
\text { Endpoint: To determine the ability } \\
\text { of Leukine }{ }^{\circledR} \text { treatment to induce } \\
\text { clinical response and remission in } \\
\text { patients who experience disease } \\
\text { relapse following initial response } \\
\text { or remission with treatment with } \\
\text { Leukine }{ }^{\circledR} \\
\text { Patient population: Patients with } \\
\text { moderately to severely active } \\
\text { Crohn's disease } \\
\mathrm{n} \approx 330\end{array}$ & Closed to enrolment & Berlex & $\begin{array}{l}\text { Anna Williford, RN } \\
\text { Louisville, Ky., USA } \\
\text { Tel.: +1 } 5025830880 \\
\text { Fax: +1 } 5025833307 \\
\text { E-Mail: } \\
\text { aowill01@gwise.louisville.edu }\end{array}$ \\
\hline $\begin{array}{l}\text { Randomized, double- } \\
\text { blind, placebo-controlled } \\
\text { phase III induction study } \\
\text { to assess the efficacy } \\
\text { and safety of } \\
\text { Sargramostim } \\
\text { (Leukine }{ }^{8} \text { ) in patients } \\
\text { with active Crohn's } \\
\text { disease (N.O.V.E.L. 8) }\end{array}$ & $\begin{array}{l}\text { Phase III, multicenter, double- } \\
\text { blind, placebo-controlled, parallel } \\
\text { group trial } \\
\text { Regimen: Initial } 6 \mu \mathrm{g} / \mathrm{kg} \text { Leu- } \\
\text { kine® administered sc once daily } \\
\text { for } 8 \text { weeks. Patients who respond } \\
\text { to } 8 \text { weeks of Leukine® treatment } \\
\text { and then relapse during the } \\
\text { treatment-free observation phase } \\
\text { will be randomized to receive } \\
\text { either } 6 \mu \mathrm{g} / \mathrm{kg} \text { Leukine }{ }^{\circledR} \text { or } \\
\text { placebo, administered sc once } \\
\text { daily for } 8 \text { weeks } \\
\text { Control: Placebo } \\
\text { Endpoint: Clinical response with } \\
\text { decrease in CDAI } \\
\text { Patient population: Patients with } \\
\text { Crohn's disease, CDAI } \geq 220 \text { and } \\
\leq 475\end{array}$ & Ongoing & Berlex & $\begin{array}{l}\text { Anna Williford, RN } \\
\text { Louisville, Ky., USA } \\
\text { Tel.: +1 } 5025830880 \\
\text { Fax: +1 } 5025833307 \\
\text { E-Mail: } \\
\text { aowill01@gwise.louisville.edu }\end{array}$ \\
\hline
\end{tabular}




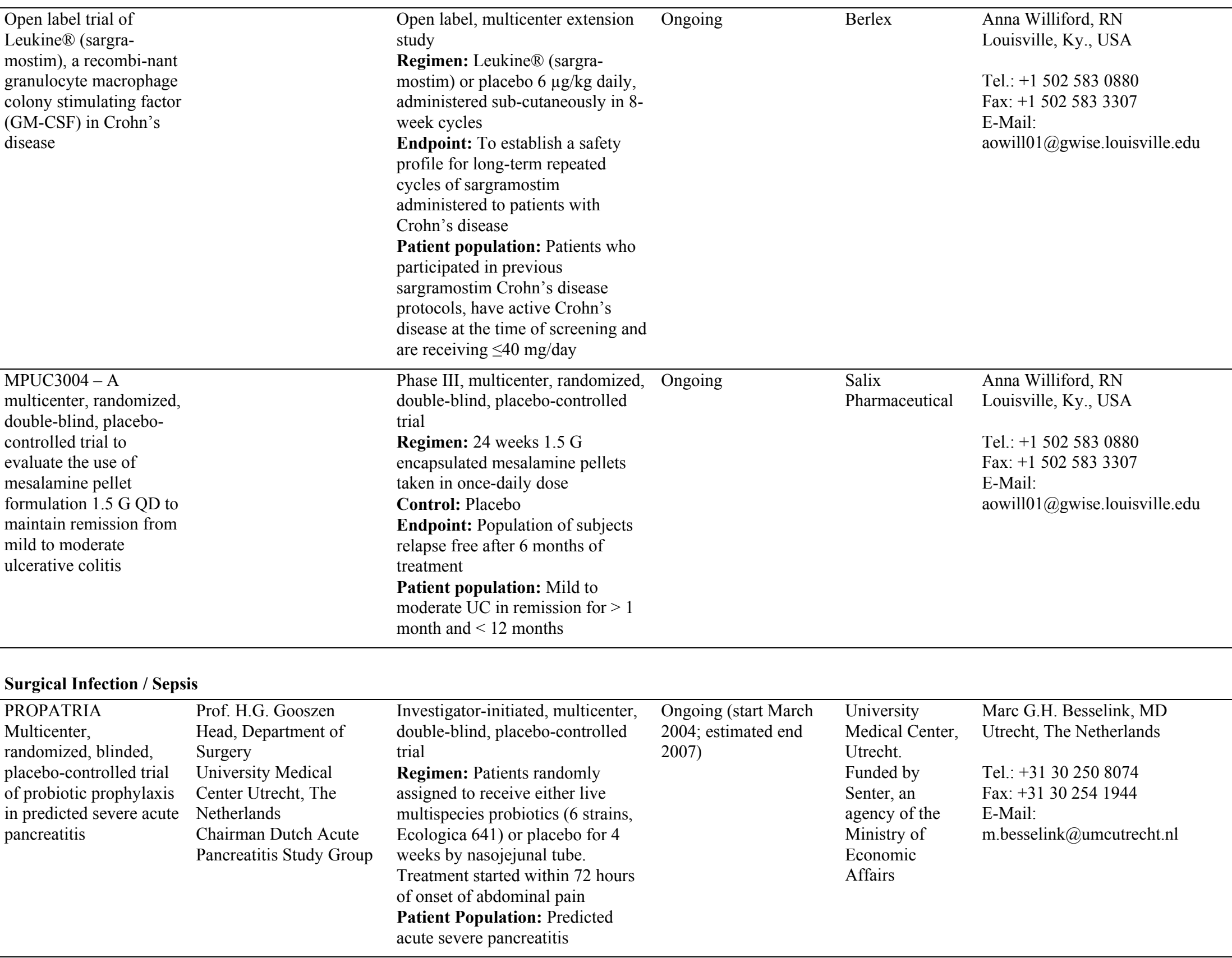




\begin{tabular}{|c|c|c|c|c|c|}
\hline $\begin{array}{l}\text { PANTER } \\
\text { Minimally invasive } \\
\text { 'step-up approach' vs. } \\
\text { maximal necrosectomy } \\
\text { in patients with acute } \\
\text { necrotizing pancreatitis }\end{array}$ & $\begin{array}{l}\text { Prof. H.G. Gooszen } \\
\text { Head, Department of } \\
\text { Surgery } \\
\text { University Medical } \\
\text { Center Utrecht, The } \\
\text { Netherlands } \\
\text { Chairman Dutch Acute } \\
\text { Pancreatitis Study Group }\end{array}$ & $\begin{array}{l}\text { Multicenter ( } 20 \text { centers of the } \\
\text { Dutch Acute Pancreatitis Study } \\
\text { Group), prospective, non-blinded, } \\
\text { active-controlled trial } \\
\text { Patients are randomized between } \\
\text { A) Maximal necrosectomy by } \\
\text { laparotomy with continuous } \\
\text { postoperative lavage and B) CT- } \\
\text { guided percutaneous or endoscopic } \\
\text { transgastric drainage, if necessary } \\
\text { followed by videoscopic assisted } \\
\text { retroperitoneal debridement } \\
\text { (VARD) } \\
\text { Endpoint: Total mortality and } \\
\text { major morbidity } \\
\text { Patient population: Patients with } \\
\text { (suspected) infected necrotizing } \\
\text { pancreatitis, preferably > days after } \\
\text { onset of symptoms } \\
n=88\end{array}$ & $\begin{array}{l}\text { Ongoing (start January } \\
\text { 2006; end January } \\
\text { 2009) } \\
\text { Manuscript of design } \\
\text { and rationale of } \\
\text { protocol submitted to } \\
\text { BioMed Central, } \\
\text { Surgery }\end{array}$ & $\begin{array}{l}\text { The Netherlands } \\
\text { Organization for } \\
\text { Health Research } \\
\text { and } \\
\text { Development } \\
\text { (ZonMw, grant } \\
\text { no. 945-06-910) }\end{array}$ & $\begin{array}{l}\text { Prof. H.G. Gooszen } \\
\text { Utrecht, The Netherlands } \\
\text { Tel.: +31 } 302509111 \\
\text { Fax: +31 } 302531944 \\
\text { E-Mail: h.gooszen@umcutrecht.nl }\end{array}$ \\
\hline \multicolumn{6}{|l|}{ Miscellaneous } \\
\hline $\begin{array}{l}\text { EUROPAC } 2 \\
\text { Double-blind } \\
\text { randomized controlled } \\
\text { trial to investigate the } \\
\text { efficacy of Antox and } \\
\text { MGCT for the treatment } \\
\text { of hereditary pancreatitis } \\
\text { and idiopathic chronic } \\
\text { pancreatitis }\end{array}$ & & $\begin{array}{l}\text { Phase III, double-blind, placebo- } \\
\text { controlled randomized trial } \\
\text { Regimen: Antioxidants for } 1 \text { year } \\
\text { Control: Placebo } \\
\text { Primary endpoint: Reduction in } \\
\text { the number of days of pancreatic } \\
\text { pain } \\
\text { Secondary endpoint: Analgesic } \\
\text { use; hospital admissions; quality of } \\
\text { life scores } \\
\text { Patient population: Patients (5-40 } \\
\text { years old) with hereditary pancre- } \\
\text { atitis or ideopathic chronic pancre- } \\
\text { atitis, registered with EUROPAC, } \\
\text { having characteristic pain that is } \\
\text { either intermittent or continuous }\end{array}$ & $\begin{array}{l}\text { Recruitment } \\
\text { (start 2006) }\end{array}$ & & $\begin{array}{l}\text { Michael G.T. Raraty, MB, BS, } \\
\text { PhD, FRCS } \\
\text { EUROPAC Study Coordinator } \\
\text { Liverpool, UK } \\
\text { Tel.: +44 } 1517064170 \\
\text { Fax: +41 } 1517065826 \\
\text { E-Mail: mraraty@liv.ac.uk / } \\
\text { europac@liv.ac.uk }\end{array}$ \\
\hline $\begin{array}{l}\text { Mesh plug versus } \\
\text { Lichtenstein, prospective } \\
\text { and randomized study }\end{array}$ & $\begin{array}{l}\text { Dr. A. Wildisen } \\
\text { Kantonales Spital Sursee- } \\
\text { Wolhusen, Switzerland }\end{array}$ & $\begin{array}{l}\text { Phase III, multicenter, prospective, } \\
\text { single-blind trial } \\
\text { Endpoint: Hernia recurrence } \\
\text { n=900 }\end{array}$ & $\begin{array}{l}\text { Ongoing } \\
\text { (end 2007) }\end{array}$ & & $\begin{array}{l}\text { Dr. A. Wildisen } \\
\text { Sursee-Wolhusen, Switzerland } \\
\text { Tel.: +41419264155 }\end{array}$ \\
\hline
\end{tabular}




\begin{tabular}{|c|c|c|c|c|c|}
\hline $\begin{array}{l}\text { POVATI: Postsurgical } \\
\text { pain outcome in patients } \\
\text { with vertical and trans- } \\
\text { verse abdominal } \\
\text { incision: A randomized } \\
\text { controlled equivalence } \\
\text { trial } \\
\text { ISRCTN No.: } 60734227\end{array}$ & $\begin{array}{l}\text { Prof. M.W. Büchler } \\
\text { Department of Surgery } \\
\text { University of Heidelberg } \\
\text { Germany }\end{array}$ & $\begin{array}{l}\text { Single center, prospective, patient- } \\
\text { blind trial } \\
\mathrm{n}=200\end{array}$ & Ongoing & $\begin{array}{l}\text { Prof. M.W. } \\
\text { Büchler }\end{array}$ & $\begin{array}{l}\text { Dr. Christoph Seiler, MSc } \\
\text { Heidelberg, Germany } \\
\text { Tel.: +496221 } 566986 \\
\text { Fax: +496221566988 } \\
\text { E-Mail: } \\
\text { christoph_seiler@med.uni- } \\
\text { heidelberg.de } \\
\text { www.sdgc.de }\end{array}$ \\
\hline $\begin{array}{l}\text { LAPCON: Total laparos- } \\
\text { copic versus conven- } \\
\text { tional ileoanal pouch } \\
\text { procedure: a randomized } \\
\text { controlled trial }\end{array}$ & $\begin{array}{l}\text { Prof. M.W. Büchler } \\
\text { Department of Surgery } \\
\text { University of Heidelberg } \\
\text { Germany }\end{array}$ & $\begin{array}{l}\text { Single center, prospective, open } \\
\text { trial } \\
\text { Control: Laparoscopic versus } \\
\text { conventional ileoanal pouch } \\
\text { procedure } \\
\text { Endpoint: Intraoperative blood } \\
\text { loss } \\
\text { Patient population: Patients with } \\
\text { familiar polyposis or ulcerative } \\
\text { colitis, scheduled for elective } \\
\text { proctocolectomy with ileoanal } \\
\text { pouch }\end{array}$ & Ongoing & $\begin{array}{l}\text { Prof. M.W. } \\
\text { Büchler }\end{array}$ & $\begin{array}{l}\text { Dr. Christoph Seiler, MSc } \\
\text { Heidelberg, Germany } \\
\text { Tel.: +49 } 6221566986 \\
\text { Fax: +496221566988 } \\
\text { E-Mail: } \\
\text { christoph_seiler@med.uni- } \\
\text { heidelberg.de } \\
\text { www.sdgc.de }\end{array}$ \\
\hline $\begin{array}{l}\text { INSECT: Interrupted or } \\
\text { continuous slowly } \\
\text { absorbable suture } \\
\text { evaluation of abdominal } \\
\text { closure techniques }\end{array}$ & $\begin{array}{l}\text { Prof. M.W. Büchler } \\
\text { Department of Surgery } \\
\text { University of Heidelberg } \\
\text { Germany }\end{array}$ & $\begin{array}{l}\text { Multicenter, prospective, open trial } \\
\text { Control: Comparison of three } \\
\text { techniques } \\
\text { Endpoint: Frequency of } \\
\text { abdominal hernias after } 12 \text { months } \\
\text { and } 3 \text { years } \\
\text { Patient population: Patients who } \\
\text { are planned to undergo an elective } \\
\text { abdominal operation }\end{array}$ & Ongoing & BBD-Aesculap & $\begin{array}{l}\text { Dr. Christoph Seiler, MSc } \\
\text { Heidelberg, Germany } \\
\text { Tel.: +496221 } 566986 \\
\text { Fax: +496221566988 } \\
\text { E-Mail: } \\
\text { christoph_seiler@med.uni- } \\
\text { heidelberg.de } \\
\text { www.sdgc.de }\end{array}$ \\
\hline $\begin{array}{l}\text { CLIVIT: Clips vs. } \\
\text { Ligatures. A multicenter } \\
\text { randomized controlled } \\
\text { trial }\end{array}$ & $\begin{array}{l}\text { Prof. M.W. Büchler } \\
\text { Department of Surgery } \\
\text { University of Heidelberg } \\
\text { Germany }\end{array}$ & $\begin{array}{l}\text { Multicenter, prospective, open trial } \\
\text { Control: Comparison of two } \\
\text { techniques to control vessels in } \\
\text { thyroid surgery } \\
\text { Endpoint: Time of resection } \\
\text { Patient population: Patients who } \\
\text { are planned to undergo an elective } \\
\text { bilateral thyroid resection }\end{array}$ & Ongoing & BBD-Aesculap & $\begin{array}{l}\text { Dr. Christoph Seiler, MSc } \\
\text { Heidelberg, Germany } \\
\text { Tel.: +49 } 6221566986 \\
\text { Fax: +496221566988 } \\
\text { E-Mail: } \\
\text { christoph_seiler@med.uni- } \\
\text { heidelberg.de } \\
\text { www.sdgc.de }\end{array}$ \\
\hline
\end{tabular}




\begin{tabular}{|c|c|c|c|c|c|}
\hline Ongoing Clinical Trials (cont & inued) & & & & \\
\hline $\begin{array}{l}\text { Open, randomized, } \\
\text { multicenter, phase IIIb } \\
\text { study for } 5 \text { years to } \\
\text { assess long-term effi- } \\
\text { ciency and tolerability of } \\
\text { esomeprazole compared } \\
\text { to laparoscopic anti- } \\
\text { reflux surgery in adult } \\
\text { subjects with chronic } \\
\text { gastroesophageal reflux } \\
\text { disease }\end{array}$ & $\begin{array}{l}\text { Lars Lundell, MD } \\
\text { Sahlgrenska University } \\
\text { Hospital } \\
\text { Gothenburg, Sweden }\end{array}$ & $\begin{array}{l}\text { Phase IIIb, multicenter ( } 60 \\
\text { centers), prospective, open trial } \\
\text { Control: Surgery } \\
\text { Regimen: } 20-40 \mathrm{mg} \text { esomeprazole } \\
\text { for } 5 \text { years } \\
\text { Endpoint: Time to treatment } \\
\text { failure } \\
\text { Patient population: Patients } \\
\text { suffering from confirmed gastro- } \\
\text { esophageal reflux disease for more } \\
\text { than } 6 \text { months } \\
n=550\end{array}$ & $\begin{array}{l}\text { Follow-up (end June } \\
\text { 2008) }\end{array}$ & Astra Zeneca & $\begin{array}{l}\text { Dr. Christoph Seiler, MSc } \\
\text { Heidelberg, Germany } \\
\text { Tel.: +496221566986 } \\
\text { Fax: +496221566988 } \\
\text { E-Mail: } \\
\text { christoph_seiler@med.uni- } \\
\text { heidelberg.de } \\
\text { www.sdgc.de }\end{array}$ \\
\hline
\end{tabular}


Digestive

surgergery

\section{Questionnaire}

\section{for Trial Submission}

To submit information on a clinical trial for publication in the 'Ongoing Clinical Trials' section, please complete this short questionnaire.

\section{1) Title of study (in full)}

\section{2) Lead investigator}

Title:

Name:

Affiliation:

\section{3) Field of study}

Please indicate one or more of the following categories:

$\square$ Oncology $\square$ Inflammatory Bowel Disease

$\square$ Motility $\square$ Surgical Infection/Sepsis

$\square$ Miscellaneous

Other (please specify):

\section{4) Study phase (drug trials only)}

Please indicate one of the following categories:

$\square$ Phase I $\square$ Phase II $\square$ Phase III $\square$ Phase IV

$\square$ Independent (investigator initiated)

\section{5) Study design}

$\square$ Multicenter $\square$ Single center (please indicate as appropriate) If multicenter, please state how many centers are involved:

$\square$ Prospective $\square$ Retrospective (please indicate as appropriate)

If prospective, level of randomization (e.g. single blind, double blind, investigator-blind):

Controls (i.e. placebo-, active-, etc.):

Cross-over design (one-way, two-way, etc.):

Duration of therapy (if applicable):

\section{KARGER}

Dosing regimen (if applicable):

Primary study endpoint:

Other information on study design (please include any other details that might be relevant):

\section{6) Patients}

Number of patients (planned):

Patient population (e.g. patients with mild UC previously controlled on...):

\section{7) Current status of study}

Start date:

Estimated end date:

Status (please indicate as appropriate):

$\begin{array}{ll}\square \text { Development } & \square \text { Recruitment } \quad \square \text { Ongoing } \\ \square \text { LPO } & \square \text { Analysis in progress }\end{array}$

$\square$ LPO $\quad \square$ Analysis in progress

\section{Publication}

Abstract submitted to:

Manuscript submitted to:

\section{8) Sponsor}

\section{9) Contact for further information:}

Name:

Address:

Telephone:

Fax:

E-Mail:

Please return the completed questionnaire as soon as possible to the Section Editor:

Susan Galandiuk, MD

Department of Surgery

School of Medicine

University of Louisville

Louisville, KY 40292, USA

Tel.: +1 5028525442

Fax: +1 5028528915 or contact:

S. Karger AG

Attn.: Ms. Yvonne Rebmann

Allschwilerstrasse 10

PO Box

CH-4009 Basel

Tel.: +41613061351

Fax: +41613061234

E-Mail: y.rebmann@karger.ch 